De Tampere à Séville : bilan de la sécurité européenne $(2 / 2)$

\title{
L'impact des événements du 11 septembre sur la création de l'espace de liberté, de sécurité, et de justice. Partie 2
}

\section{Pierre Berthelet}

\section{(2) OpenEdition \\ Journals}

\section{Édition électronique}

URL : http://journals.openedition.org/conflits/799

DOI : $10.4000 /$ conflits.799

ISSN : $1777-5345$

Éditeur :

CCLS - Centre d'études sur les conflits lilberté et sécurité, L'Harmattan

Édition imprimée

Date de publication : 1 juin 2002

ISBN : 2-7475-3030-2

ISSN : 1157-996X

Référence électronique

Pierre Berthelet, «L'impact des événements du 11 septembre sur la création de l'espace de liberté, de sécurité, et de justice. Partie 2 », Cultures \& Conflits [En ligne], 46 | été 2002, mis en ligne le, consulté le 30 mars 2021. URL : http://journals.openedition.org/conflits/799 ; DOI : https://doi.org/10.4000/ conflits.799

Ce document a été généré automatiquement le 30 mars 2021.

Creative Commons License 


\title{
L'impact des événements du 11 septembre sur la création de l'espace de liberté, de sécurité, et de justice. Partie 2
}

\author{
Pierre Berthelet
}

Le renforcement de l'action extérieure de l'Union en matière de lutte anti-terroriste Le combat contre le terrorisme, du fait de sa dimension internationale, nécessite une collaboration sans faille entre tous les Etats. L'illustration de l'implantation du réseau Al-Quaïda dans un pays isolé diplomatiquement en raison de son régime islamique très dur, oblige la Communauté internationale à développer une très forte solidarité pour éviter qu'un pays mis au ban des nations ne se transforme en un sanctuaire pour le terrorisme.

L'Union soutient les Etats-Unis dans la lutte contre le terrorisme. Le dialogue s'est nettement renforcé en particulier en matière d'entraide policière et judiciaire.

La lutte contre le terrorisme a également lieu avec d'autres partenaires. Cette coopération se traduit notamment par l'inscription de clauses anti-terrorisme dans les accords signés entre la Communauté européenne et les pays tiers.

Une meilleure collaboration avec les Etats-Unis

La coopération entre l'Union européenne et les Etats-Unis a connu un saut qualitatif. Après les attentats du 11 septembre, les liens avec l'Union européenne se sont fortement resserrés.

Le premier effet de l'approfondissement des relations se traduit par une amélioration significative de l'échange de renseignements concernant les organisations terroristes. Les Etats-Unis se sont montrés disposés à coopérer à ce sujet. Ils exercent même une pression très forte sur les Etats membres pour obtenir les informations nécessaires.

En dehors de l'augmentation des flux de renseignements, la coopération avec les EtatsUnis en matière de lutte contre le terrorisme se concrétise principalement par $^{1}$ : 
- La coopération entre Eurojust et les Etats-Unis. Le rapport d'activité 2001 d'Eurojust ${ }^{2}$ précise que les conclusions du Conseil JAI du 20 septembre 2001 ont confié à l'Unité provisoire Eurojust un mandat spécifique pour étudier et développer des relations ainsi que des arrangements de travail avec les Etats-Unis d'Amérique sur les questions de terrorisme, l'idée étant de renforcer l'entraide judiciaire par le biais d'Eurojust. Pour donner suite à ces conclusions, l'Unité s'est réunie à Bruxelles avec des membres de la Mission des Etats-Unis auprès de l'Union européenne. Les Etats-Unis ont désigné leurs magistrats de liaison basés à Paris comme responsables spécifiques des relations avec l'Eurojust provisoire, et ultérieurement, avec l'unité Eurojust définitive. Un haut responsable du ministère américain de la justice a été désigné pour être le point de contact à Washington pour les relations avec l'Unité provisoire. Depuis lors, des réunions régulières ont lieu entre Eurojust et les autorités américaines.

- La coopération entre Europol et les Etats-Unis. Le 6 décembre 2001, un accord ${ }^{3}$ a été signé entre le directeur d'Europol et les Etats-Unis afin de permettre l'échange de données sur la criminalité entre l'office et l'Administration américaine. Les informations portent notamment sur le terrorisme et le blanchiment de capitaux. Il ne s'agit toutefois pas de transmettre des données à caractère personnel mais d'échanger des meilleures pratiques, des données sur l'évolution de la criminalité et des programmes communs de formation. Pour les données à caractère personnel, un accord spécifique est actuellement en cours de négociation entre Europol et les EtatsUnis. L'accord signé le 6 décembre prévoit également un échange d'officiers de liaison. A cet effet, deux officiers d'Europol ont été désignés à Washington.

- Le projet d'accord d'entraide judiciaire. Après plusieurs contacts exploratoires, les Etats membres et les Etats-Unis ont accepté le principe de la conclusion d'un accord d'entraide judiciaire. Cet accord, fondé sur l'art. 38 TUE, vise à renforcer la coopération judiciaire pénale, tant d'un point de vue de l'extradition qu'en ce qui concerne l'entraide mineure. Les Etats membres ont donné à la présidence espagnole un mandat de négociation avec les Etats-Unis au cours du Conseil «Justice, affaires intérieures et protection civile » des 25 et 26 avril 2002. Les discussions promettent d'être intenses car les Etats membres tiennent à inclure toute une série de sauvegardes jugées sensibles. Un accord devra être trouvé sur la peine de mort, la perpétuité réelle, les procédures spéciales, la protection des données personnelles et le rapport entre ce futur accord et les différents accords bilatéraux.

Les futurs traités signés avec les autres pays tiers concernent des thèmes plus généraux comme la libre circulation des biens et des services mais l'on observe depuis les attentats du 11 septembre, l'inscription de clauses anti-terrorisme dans ces accords.

L'insertion de clauses anti-terrorisme avec les pays tiers

A l'instar des clauses anti-blanchiment ou des clauses anti-drogue, le Conseil a souhaité qu'une clause soit inscrite pour combattre le terrorisme dans chaque nouvel accord signé entre la Communauté et un pays tiers.

Conformément aux conclusions du Comité politique et de sécurité du 11 décembre 2001, le Secrétariat général du Conseil, en liaison avec la Commission, a diffusé un document d'orientation sur l'évaluation des relations de l'UE avec les pays tiers en matière de lutte contre le terrorisme. L'une des orientations proposées dans ce 
document concerne l'insertion d'une clause anti-terrorisme dans les accords avec ces pays, cette clause pouvant être insérée dans l'accord lui-même, faire partie d'un échange de lettres entre les parties, ou être intégrée dans une déclaration jointe à l'accord.

Une première clause figure dans l'accord d'association entre la Communauté et l'Algérie. Cet accord a été signé à Valence le 22 avril 2002 en marge de la Conférence euro-méditerrannée. L'art. 90 prévoit le renforcement de la lutte anti-terrorisme entre les deux parties par:

- la mise en œuvre intégrale de la résolution 1373 du Conseil de sécurité,

- un échange d'informations sur les groupes anti-terroristes,

- un échange d'expériences sur les moyens et les méthodes pour lutter contre le terrorisme ${ }^{4}$.

La lutte anti-terroriste fait aussi l'objet d'accords spécifiques. L'accord euroméditerrannéen avec le Liban a été signé le 17 juin 2002 et un autre accord, sous forme d'échange de lettres, porte sur la coopération en matière de lutte anti-terroriste. Par ailleurs, les ministres des affaires étrangères sont parvenus le même jour à un accord politique concernant le mandat à donner à la Commission pour négocier un accord sur la lutte contre le terrorisme avec l'Iran. L'insertion de clauses antiterrorisme avec les pays tiers est inscrite dans la «feuille de route » anti-terrorisme. Il s'agit du 11e objectif.

La création d'une « feuille de route » anti-terrorisme

La création d'une «feuille de route » apporte des avantages appréciables en terme de cohérence de l'action des Etats membres en matière de terrorisme. Cependant, elle n'est pas sans inconvénients.

Les avantages de l'instauration d'une "feuille de route"

La « feuille de route » vise à assurer une meilleure coordination de l'activité du Conseil en matière de lutte contre le terrorisme. Elle reprend l'ensemble des mesures et des initiatives à mettre en œuvre dans le cadre du plan d'action initié par le Conseil européen du 21 septembre 2001, décidé suite aux événements du 11 septembre 2001. La feuille de route fait état des travaux dans les différentes formations du Conseil. Elle regroupe les différents points par secteurs relevant des Conseils "Affaires générales ", " Justice et affaires intérieures », « ECOFIN » et « Transports ».

La présidence belge puis espagnole, réalise une mise à jour mensuelle du document ${ }^{5}$. Le texte contient 68 mesures à réaliser. Les objectifs sont très hétérogènes. Ils vont du « renforcement des relations avec certains pays d'Asie particulièrement concernés par la situation actuelle » à la "création d'équipes communes d'enquêtes » en passant par "l'extension des mécanismes d'échanges d'informations automatiques entre les cellules de renseignement financier » et l'adoption de "mesures additionnelles de sûreté dans l'aviation civile $»^{6}$.

Le document, sous forme de tableau, détermine les moyens et les objectifs pour y parvenir, le délai et les responsables. Il fait état des progrès déjà réalisés et annonce les travaux à venir. 
L'adoption de la feuille de route apporte davantage de cohérence dans l'action des Etats membres en matière de lutte contre le terrorisme :

- d'abord elle assure une meilleure rationalisation de leur activité en désignant l'acteur devant accomplir telle ou telle mesure. Autrement dit, la feuille de route définit « qui est responsable de quoi ».

- ensuite elle maintient la dynamique générée par les conclusions du Conseil européen. Elle a vocation à déterminer la distance déjà couverte et celle qui reste encore à parcourir. Sa mise à jour régulière met en exergue les actions accomplies et celles devant encore l'être.

- enfin, elle fait apparaître les échéances qui n'ont pas été respectées. L'existence d'un calendrier précis décrivant la date à laquelle les mesures doivent être adoptées, prévient tout dépassement des délais prévus.

Ses inconvénients

La création d'une feuille de route consacrée à la lutte contre le terrorisme n'est pas sans désavantages. Elle contribue en effet à l'inflation des plans d'action concernant le domaine de la justice et des affaires intérieures.

Il existe déjà le "Scoreboard ". Sur demande des chefs d'Etat lors de sa réunion de Tampere des 15 et 16 octobre 1999, la Commission a présenté un "Tableau de bord » destiné à déterminer les mesures à prendre pour réaliser un espace de liberté, de sécurité et de justice et évaluer les progrès effectués à cette fin. Il est mis à jour tous les semestres.

Il convient également de citer le plan d'action en matière de lutte contre la drogue 2000-2004 adopté en 19997. Ce plan, approuvé par le Conseil européen d'Helsinki au mois de décembre 1999, a pour mission de développer une stratégie globale, pluridisciplinaire et intégrée de lutte contre la drogue. Il fait suite au plan d'action de lutte contre la drogue (1994-1999) adopté par le Conseil le 2 juin $1995^{\circ}$.

Il faut aussi mentionner la "stratégie de l'Union européenne sur la prévention et le contrôle de la criminalité organisée" adoptée par le Conseil "Justice, affaires intérieures et protection civile » du 27 mars $2000^{9}$.

Le Conseil a enfin adopté le 28 février 2002 le plan d'action global de la présidence espagnole relatif à la lutte contre l'immigration clandestine ${ }^{10}$. Ce document propose tout un ensemble de mesures dont les principales d'entre elles concernent la mise en place d'un système d'alerte avancé concernant l'immigration clandestine, la promotion des accords de réadmission et l'harmonisation des normes communes de rapatriement, l'instauration d'une police européenne des frontières, et la confiscation des produits issus de la traite et l'octroi de bénéfices aux victimes disposées à coopérer.

La «feuille de route» sera une strate supplémentaire constituée par ces différents plans d'action. Leur prolifération nuit donc à la visibilité de l'action du Conseil. Ils sont destinés à organiser l'action des Etats membres, or, leur multiplication est susceptible d'avoir l'effet inverse de celui visé. En outre, cette profusion n'est pas le garant du respect des mesures entreprises.

La mise en place d'une "feuille de route" a servi cependant de guide concernant l'élaboration de nouveaux instruments normatifs. 
L'élaboration de nouveaux instruments normatifs

Les événements du 11 septembre ont contraint les Etats membres à identifier tous les moyens dont ils disposent (ou en projet). C'est le cas, on l'a vu précédemment, du mandat d'arrêt européen ou d'Eurojust ${ }^{11}$.En l'espace de quelques mois seulement, le Conseil a permis à l'Union de se doter d'une législation anti-terroriste par le biais d'une décision-cadre.

Les évènements du 11 septembre ont contribué à la mise sur pied de projets spécifiques destinés à combattre le terrorisme.

Ils ont aussi permis d'accélérer les projets moins spécifiques à la lutte contre ce fléau mais susceptibles néanmoins d'être utiles.

La décision-cadre relative à la lutte contre le terrorisme

Pivot du dispositif, la décision-cadre relative à la lutte contre le terrorisme a pour objectif d'harmoniser les infractions pénales en la matière.

Le texte donne une définition de l'acte de terrorisme et du groupe terroriste. Il faut dire que la situation des Etats membres de l'Union européenne est différente d'un point de vue juridique ${ }^{12}$. C'est pourquoi, la Commission, dans une proposition présentée le 19 septembre $2001^{13}$, suggère aux Etats de se doter d'une législation commune relative au terrorisme.

Cette proposition se fonde sur l'article 31, point e) stipulant que l'action en commun dans le domaine de la coopération judiciaire en matière pénale vise à adopter progressivement des mesures instaurant des règles minimales relatives aux éléments constitutifs des infractions pénales et aux sanctions applicables dans le domaine notamment du terrorisme.

La décision-cadre ${ }^{14}$ telle qu'adoptée par les Ministres réunis au sein du Conseil « Justice, affaires intérieures et protection civile » du 13 et 14 juin 2002 définit l'infraction de terrorisme comme étant les actes intentionnels consistant à "gravement intimider une population, ou contraindre indûment des pouvoirs publics ou une organisation internationale à accomplir ou à s'abstenir d'accomplir un acte quelconque, ou gravement déstabiliser ou détruire les structures fondamentales politiques, constitutionnelles, économiques ou sociales d'un pays ou une organisation internationale;

a) les atteintes contre la vie d'une personne pouvant entraîner la mort ;

b) les atteintes graves à l'intégrité physique d'une personne ;

c) l'enlèvement ou la prise d'otage $»^{15}$.

En ce qui concerne la définition du but terroriste, les débats ont porté sur l'équilibre à trouver entre la nécessité de réprimer efficacement les infractions terroristes et de garantir les libertés et droits fondamentaux afin d'assurer que des actions légitimes,

par exemple dans le cadre d'activités syndicales ou de mouvements

anti-mondialisation, ne puissent en aucun cas entrer dans son champ d'application.

La décision-cadre prévoit que chaque Etat membre prend les mesures nécessaires pour que les infractions relatives au terrorisme soient passibles de sanctions pénales effectives, proportionnées et dissuasives, susceptibles d'entraîner l'extradition.

Les Etats membres doivent aussi adopter en vertu de cette décision-cadre les mesures nécessaires pour que les infractions de terrorisme soient passibles de peines privatives de liberté maximales ne pouvant être inférieures à quinze ans pour la direction d'un 
groupe terroriste, et à huit ans pour la participation aux activités d'un groupe terroriste.

Le texte prévoit aussi de rendre punissable l'incitation, la complicité, et la tentative.

Le combat contre le terrorisme a permis d'initier d'autres projets spécifiques.

L'émergence de nouveaux projets spécifiques à la lutte anti-terroriste

De nouvelles initiatives ont vu le jour principalement sous présidence espagnole. Un des instruments qui constitue la pierre angulaire du dispositif anti-terroriste est le règlement 467/2001 destiné à geler les avoirs des organisations terroristes sur lequel il convient de s'attarder plus longuement.

La présidence espagnole a présenté tout un ensemble de mesures destinées à combattre ce phénomène. En voici trois illustrations :

- L'instauration d'un formulaire type destiné aux échanges d'informations concernant les incidents provoqués par des groupes radicaux violents liés au terrorisme.

Un projet de décision ${ }^{16}$ a été présenté au "groupe terrorisme » du Conseil par la présidence espagnole. Le formulaire contenu dans ce projet est destiné à mieux prévenir et à réprimer le radicalisme violent des jeunes en milieu urbain. Il concerne seulement des personnes ayant des antécédents judiciaires liés au terrorisme. Le texte de la présidence précise néanmoins que tout pays qui le souhaite pourra décider l'échange d'informations à d'autres personnes. Il insiste sur le fait que l'initiative ne vise pas les personnes exerçant leurs droits et leurs libertés de réunion, de manifestation ou d'expression.

- La constitution d'équipes multinationales d'enquête.

Ce projet de recommandation ${ }^{17}$ du Conseil suggère de constituer au cas par cas des équipes composées de représentants de différents Etats membres en vue notamment de la collecte et de l'échange d'informations utiles à la lutte contre le terrorisme dans le cadre d'opérations concrètes et dans n'importe quel pays de l'Union européenne. Ces équipes seraient composées d'experts des services de sécurité ou de police chargés de la lutte contre le terrorisme dans l'Union européenne et assistés, le cas échéant, par des fonctionnaires d'Europol. Le projet de recommandation souligne que les équipes multinationales d'enquête ne doivent pas être confondues avec les équipes communes d'enquêtes prévues par la Convention européenne d'entraide judiciaire en matière pénale, étant donné que ces actions se déroulent dans des champs d'application totalement distincts.

- Le renforcement de l'entraide policière et judiciaire contre les personnes figurant sur la liste des organisations terroristes

L'Espagne a présenté le 18 mars une initiative ${ }^{18}$ visant à renforcer la coopération pénale impliquant les personnes, groupes ou entités figurant sur la liste des organisations ou des personnes appartenant ou liées à l'ancien régime taliban ou au réseau terroriste $\mathrm{Al}-$ Qaïda dont les avoirs doivent être gelés. Le texte prévoit la création d'un point de contact au sein des services policiers et judiciaires ayant accès aux informations concernant les enquêtes pénales en ce qui concerne les infractions terroristes. Il incite aussi les Etats membres à prendre les mesures nécessaires pour que les demandes d'entraide et les demandes de reconnaissance des décisions judiciaires en relation avec 
des infractions terroristes soient traitées d'urgence et en priorité. Il leur demande aussi à veiller à ce que tout moyen de preuve puisse être immédiatement accessible aux autorités d'autres Etats membres.

Cette initiative est fondée sur la position commune 2001/931/PESC à l'instar du règlement destiné à geler les avoirs des organisations terroristes.

Le Conseil a adopté le 27 décembre 2001 un règlement ${ }^{19}$ permettant le gel des avoirs des organisations et d'individus terroristes. Il comprend différentes définitions tirées des accords internationaux existants, particulièrement la convention internationale pour la répression du financement du terrorisme. Par ailleurs, il prévoit la définition d'une liste régulièrement mise à jour des organisations et individus qui doivent être considérés par les quinze Etats membres comme terroristes. Le texte précise aussi que les Etats membres se prêtent mutuellement, et prêtent aux Etats tiers la plus grande assistance lors des enquêtes criminelles.

Le Conseil a adopté également le 27 décembre une décision ${ }^{20}$ contenant une liste de douze organisations et de trente individus qui doivent être considérés comme terroristes par les quinze Etats membres. Ce texte, adopté en vertu du règlement précité, mentionne différents groupes comme l'ETA, le Jihad islamique palestinien et le Hamas.

Le règlement du 27 décembre 2001 élargit le dispositif de sanction à l'ensemble des organisations et d'individus terroristes. Déjà, le Conseil avait adopté le 6 mars 2001 un texte $^{21}$ destiné à geler les fonds des Talibans.

Mettant en œuvre la résolution 1390 du 16 janvier 2002 du Conseil de Sécurité de l'ONU, le Conseil a adopté le 27 mai 2002 un nouveau règlement ${ }^{22}$ modifiant le régime de sanction pour tenir compte de la chute du régime taliban et la mise en place du nouveau gouvernement. Le règlement prévoit l'abrogation de l'ancien règlement467/2001 et renforce les sanctions infligées aux entités liées à Oussama BEN LADEN en incluant toutes les "ressources économiques » et non plus seulement les capitaux et fonds financiers.

Les attentats contre les tours du WTC à New-York ont aussi permis l'accélération des projets visant à améliorer la coopération judiciaire pénale.

La naissance rapide des nouveaux instruments juridiques atteste que les Etats ont fait de la lutte contre le terrorisme leur priorité. En analysant de plus près pourquoi, on découvre que cette menace est au cœur du concept européen de sécurité.

La modification du concept européen de sécurité

Le concept européen de sécurité est créé en réaction à une menace, réelle ou imaginaire. Par conséquent, pour définir un concept européen de sécurité, il convient préalablement de déterminer quel type de menace pèse sur les Etats membres. Depuis le 11 septembre, les choses ont évolué. La menace est différente. Toutefois, elle est incarnée par le même individu.

Le concept européen de sécurité avant les attentats du 11 septembre : l'étranger membre d'une organisation criminelle

Les Etats membres se sont efforcés de trouver un ennemi commun. Ils y sont parvenus. Il s'agit de la criminalité organisée. Cette menace est personnifiée par l'étranger.

La politique de sécurité européenne actuelle trouve ses racines au milieu des années 1970 dans la peur du «terrorisme rouge ». Les phénomènes d'insécurité qui s'étaient développés à cette époque comme les attentats terroristes et les prises d'otage étaient 
analysés comme une intensification des conflits idéologiques ${ }^{23}$. Les européens avaient l'impression d'être confrontés à des problèmes identiques. Ils y voyaient un même adversaire : l'Union Soviétique.

Après la disparition du bloc de l'Est, les théoriciens qui avaient perdu leur adversaire traditionnel, ont cherché un nouvel ennemi qui menacerait l'Europe. Les militairesvont redéfinir leur objectif de protection du territoire en rencontrant celui des policiers. Le nouveau concept de sécurité doit décrire l'ennemi comme venu de l'extérieur pour menacer l'espace intérieur. Cette pénétration de la géopolitique dans la sphère de la sécurité intérieure permet de retrouver un ennemi et de redonner ainsi un sens global à tous les événements mondiaux ${ }^{24}$.

Cela dit, la détermination de cet ennemi s'est faite progressivement. Finalement, la criminalité organisée a été trouvée comme concept mobilisateur. Cet ennemi toujours identifiable, mais (pratiquement) jamais identifié, peut se cacher derrière chaque méfait. Son caractère mystérieux et impalpable, donne le sentiment d'être à la fois partout et nulle part. La criminalité organisée, en franchissant les frontières nationales et acquérant une dimension internationale, devient l'ennemi de l'intérieur et de l'extérieur.

Elle requiert une réponse forte qui exige la mise à disposition de tous les moyens dont dispose l'Union pour la combattre. La menace du crime organisé devient un justificatif à une intensification de l'action des Etats membres. C'est ainsi que tous les nouveaux actes juridiques pris en matière de la coopération policière et judiciaire l'ont été dans ce contexte ${ }^{25}$.

Cependant, cette menace est trop abstraite. Pour l'ancrer dans l'inconscient collectif, il faut la personnifier, et cette personnification a lieu dans le chef de l'immigrant.

La criminalité organisée qui est décrite comme l'ennemi commun, doit être incarnée par les personnes physiques qui en constituent le vecteur humain ${ }^{26}$. Ces personnes doivent posséder les mêmes caractéristiques que celle-ci. L'immigrant représente l'archétype de l'ennemi idéal. Il vient de l'extérieur de l'Union pour s'intégrer dans la société européenne.

La combinaison de la criminalité et de l'immigration fait porter un regard particulier sur certains groupes d'étrangers. Ces groupes minoritaires, déjà présents sur le territoire, serviraient de relais dans la stratégie d'infiltration de l'Europe du crime organisé. Concrètement, l'implantation des mafias dans l'Union européenne a lieu par le parasitage des communautés de compatriotes. Elles utilisent les immigrants déjà installés dans l'Union pour permettre leur enracinement puis leur développement dans l'Union ${ }^{27}$. "Contraintes ou complices, elles [ces diasporas] relaient les filières d'immigration illicite; dissimulent en leur sein les criminels eux-mêmes, parmi les clandestins ${ }^{28}$.

L'invasion criminelle viendrait se superposer à l'invasion migratoire. La médiatisation de clandestins abandonnés à leur sort par des trafiquants, arrivant par mer sur les côtes européennes stigmatise les peurs d'une invasion à grande échelle.

Par conséquent, la création d'une menace réunissant la criminalité organisée et l'immigration permet de justifier un concept de sécurité intérieure très large. Ce concept englobe ainsi toutes les actions relatives à la lutte contre la criminalité et contre l'immigration.

Cette théorie fournit la clef qui permet d'expliquer l'action des Etats en matière d'asile et d'immigration. "Comme il parait difficile de convaincre les Etats par les moyens mis en 
cuvre pour la lutte contre le terrorisme et la grande criminalité, il reste la possibilité de trouver de nouveaux agents d'insécurité, voire de nouveaux criminels. La criminalisation de l'étranger, connue dans les Etats, en serait renforcée par la gestion des politiques migratoires par des ensembles d'Etats $»^{29}$. Les attentats du 11 septembre sont venus modifier le concept de sécurité.

Le concept européen de sécurité après les attentats du 11 septembre : l'étranger membre d'une organisation terroriste

Le concept de sécurité jadis axé sur la criminalité organisée a été réorienté vers le terrorisme. Cependant, il n'est pas possible de parler de révolution mais d'évolution du concept. En effet, la menace même si elle a changé d'objet, demeure toujours personnifiée par l'étranger. La création du nouveau concept européen de sécurité ne contredit pas l'ancien: l'étranger demeure toujours une menace. Seulement au lieu d'être un criminel potentiel, il est un terroriste potentiel.

Les attentats du 11 septembre ont entraîné une nouvelle redéfinition de la menace. Sous l'impulsion des américains, les européens ont concentré leurs efforts vers la lutte contre les groupes terroristes et en particulier l'organisation Al-Quaïda. Les enceintes policières dans lesquelles s'échangent les informations dans ce domaine ont été réactivées. On l'a vu pour le groupe « terrorisme » du Conseil.

Les moyens alloués pour combattre ce phénomène ont été revus à la hausse. Par exemple, les Etats membres ont décidé d'augmenter le budget d'Europol de 49,5\% dont le montant initial s'élevait de 48,5 millions euros. Ces nouveaux revenus pour l'office sont consacrés à la lutte contre le terrorisme et destinés principalement au financement du système d'information d'Europol et à celui des officiers de liaison Europol à Interpol et aux USA.

Plus généralement, la lutte contre le terrorisme donne une nouvelle grille de lecture de l'action des Etats membres dans le domaine de la justice et des affaires intérieures. Ainsi, l'extension des mécanismes d'échange d'informations entre les cellules de renseignement financiers (CRF) devient un outil de lutte contre le terrorisme. Tout est vu sous le prisme de cette menace en lisant la « feuille de route " comme par exemple l'art. 2.2 de la Convention d'application Schengen qui prévoit le rétablissement des contrôles aux frontières entre les Etats au sein de l'espace Schengen. Les Etats membres ont analysé les hypothèses d'un recours coordonné à l'art. 2.2 en particulier en cas de menace terroriste.

La lutte contre le terrorisme devient également un prétexte pour limiter certains droits. Ainsi, dans une résolution votée le 14 mars 2002 en assemblée plénière à Strasbourg, les députés ont fait part de leur inquiétude concernant l'opacité dans l'adoption des règles internes par le Conseil et de la Commission. Ils se sont dit préoccupés par la décision du Conseil de classer confidentiels les ordres du jour des réunions du groupe de haut niveau et du groupe de travail UE/Etats-Unis au motif que les autorités nord-américaines s'opposent à leur publication parce qu'il s'agirait de documents de gouvernement à gouvernement.

Cette focalisation autour de la menace terroriste est dangereuse car elle satellise d'autres concepts qui n'ont pas forcément de corrélation. C'est le cas par exemple de l'immigration. 
L'architecture du concept de sécurité est modifiée mais il n'est pas altéré dans sa substance. Autrement dit, la grille de lecture est toujours identique: l'étranger demeure une menace pour la sécurité européenne.

Les attentats contre les tours du WTC ont contribué à donner un "tour de vis sécuritaire » à l 'édification de l'espace de liberté, de sécurité et de justice. En analysant les mesures initiées ou adoptées après le 11 septembre, on découvre aisément qu'elles sont axées essentiellement sur le renforcement de la coopération contre l'immigration clandestine.

En effet, l'immigration clandestine est par nature, une immigration incontrôlée et, parmi le flot des immigrants clandestins, sont susceptibles de se cacher des terroristes.

Pour y faire face, les Etats membres ont adopté très récemment toute une série de mesures comme par exemple le plan d'action global destiné à lutter contre l'immigration clandestine du 28 février 2002 ou les conclusions relatives à l'immigration clandestine par voie maritime ${ }^{30}$, dans lesquelles il demande de renforcer la coordination entre les officiers de liaison en poste dans les pays d'origine et de transit des immigrants clandestins. Il prend note que la Commission prépare une étude de faisabilité sur l'amélioration des contrôles aux frontières maritimes. Il demande de promouvoir et de développer des mesures préventives en coopération avec les pays tiers d'embarquement, de départ et de transit et d'examiner « des mesures visant les Etats tiers qui refusent de coopérer avec l'Union européenne dans la lutte contre l'immigration illégale».

La lutte contre l'immigration clandestine ne s'articule pas uniquement sur la menace terroriste. Il serait présomptueux d'affirmer que les attentats du 11 septembre justifient l'ensemble des actions menées par les Etats membres dans ce domaine. Il n'empêche que le risque d'une infiltration terroriste par l'entremise de l'immigration clandestine est une préoccupation réelle et qu'elle sous-tend les mesures développées contre l'immigration clandestine.

La lutte contre le terrorisme est également une raison supplémentaire de restreindre la politique d'asile. Une très grande suspicion prévaut envers les candidats au refuge. Après les attentats du 11 septembre, l'état d'esprit des Etats membres est qu'en cas de doute, il vaut mieux débouter un demandeur d'asile en dépit du fait qu'il ait fait l'objet de réelles persécutions plutôt que d'accorder le statut de réfugié à un étranger terroriste.

Enfin, la lutte contre le terrorisme constitue un motif supplémentaire pour renforcer les contrôles aux frontières extérieures. L'Union doit empêcher ses ennemis de s'infiltrer en Europe et elle peut le faire en renforçant ses frontières extérieures.En contrôlant les frontières, on contrôle le terrorisme et les personnes qui véhiculent le terrorisme.

La politique sécuritaire joue aussi sur les peurs qui permet de fédérer les Etats de l'Union autour de la lutte contre l'ennemi commun qu'est le terrorisme. Pour enrayer une "Europe passoire ", on érige des frontières extérieures afin de se prémunir des dangers du monde extérieur. La menace terroriste devient donc un moyen supplémentaire d'établir des frontières étanches et sûres pour protéger sa sécurité et celle de ses citoyens.

L'impact des événements sur la coopération pénale est considérable. Il l'a été au niveau des esprits, les attentats ont été très traumatisants pour l'opinion publique ${ }^{31}$. Il l'a été 
aussi au niveau de l'action politique. Les attentats contre les tours du WTC ont servi de catalyseur à la naissance de nouveaux projets comme la décision-cadre relative au terrorisme ou l'accélération de projets existants comme Eurojust.

Les effets des attentats ont été cyniquement parlant, une "bonne " chose dans la construction de l'espace de liberté, de sécurité et de justice. L'amélioration de la qualité des informations transmises à Europol en est un très bonne illustration.

Ils ont aussi servi de bouée de sauvetage à la méthode intergouvernementale utilisée dans le troisième pilier. Certains commentateurs jugent que cette méthode "malgré tous ses défauts, n'empêche pas la progression de l'intégration européenne: Europol, Eurojust, le mandat d'arrêt, la reconnaissance mutuelle en matière pénale ou civile sont autant de thèmes qui auraient été impossibles à aborder de front il y a quelques années encore ${ }^{32}$.

Quid sans les événements du 11 septembre ? Serait-on parvenu aussi vite à la création d'un mandat d'arrêt européen? Il est indéniable que les attentats ont mis la pression sur les gouvernants des Etats membres pour rassurer l'opinion publique. Il fallait faire un geste envers elle en montrant que les mesures nécessaires pour protéger les citoyens européens contre la menace terroriste étaient prises tant à l'échelle nationale qu'européenne.

Le renforcement de la coopération pénale s'est donc avéré être un gage des efforts entrepris pour assurer la sécurité des biens et des personnes. Il est une démonstration des travaux entrepris pour permettre une meilleure visibilité de l'espace de liberté, de sécurité et de justice : en adoptant toute une série de mesures pour concrétiser cet espace, les Etats membres montrent que l'objectif de l'Union de se doter d'un espace de liberté, de sécurité et de justice n'est pas un vain mot.

Face à un danger extérieur, les Etats ont su montrer qu'ils savent faire preuve de cohésion en dépassant les clivages qui les opposent. C'est le cas par exemple du mandat d'arrêt européen. Il est évident que la mise sur pied d'un tel mandat demande plusieurs mois. En revanche, la démonstration des efforts apportés pour aboutir à un règlement rapide des différences de vues pour parvenir à un accord dans les plus brefs délais, revêt une portée symbolique très forte. En s'accordant sur le texte en moins de quatre mois, les Etats membres ont exprimé clairement leur volonté de faire table rase de l'ancien mécanisme archaïque qu'est l'extradition. Il est vrai que sans les événements du 11 septembre, les Etats seraient vraisemblablement parvenus aux même résultats mais dans un laps de temps beaucoup plus long, la période de gestation des textes ayant été beaucoup plus importante ${ }^{33}$. D'ailleurs, les textes entrant dans le cadre de l'élaboration d'un espace de liberté, de sécurité et de justice mais n'ayant pas de liens avec la lutte contre le terrorisme, comme la directive relative au regroupement familial ${ }^{34}$ ou concernant la lutte contre l'exploitation sexuelle des enfants et la pédopornographie ${ }^{35}$, textes qui étaient en discussion au moment des attentats, font toujours actuellement l'objet de négociation.

Les événements du 11 septembre ont aussi démontré les difficultés institutionnelles dont souffre le troisième pilier. On a assisté à une divergence entre le Conseil «Justice, affaires intérieures et protection civile » et le Conseil européen. Les Etats membres au sein du Conseil européen sont parvenus à un consensus sur les grandes lignes, laissant le soin aux Ministres au sein des Conseils spécialisés de s'entendre sur les détails. Or, le Conseil «Justice, affaires intérieures et protection civile" n'est pas parvenu à un accord malgré les efforts de la présidence. On l'a vu pour le mandat d'arrêt européen, les ministres de certains Etats membres ont montré à plusieurs reprises des résistances 
qu'il a fallu vaincre en invoquant à nouveau le dossier au Conseil européen. Cette instance servant à l'origine d'impulsion au processus s'est transformée en instance d'appel en cas de divergence au Conseil, ce qui n'est pas sa vocation.

Maintenant le vent du 11 septembre s'est-il essoufflé? Il semble que oui: les négociations pour faire avancer le projet de décision-cadre relative à l'exécution des sanctions mutuelles ${ }^{36}$ sont difficiles. Les Etats peinent à s'accorder sur l'étendue de l'exigence de la double incrimination. L'idée était de calquer le dispositif sur les solutions dégagées concernant le mandat d'arrêt européen. Cette solution a l'avantage de la facilité et de la cohérence : tous les instruments sont soumis à un régime juridique équivalent. Il n'empêche que certains Etats rechignent bloquant de fait une prochaine mise en application du mécanisme de la reconnaissance mutuelle.

En revanche, les événements du 11 septembre ont enraciné la lutte contre le terrorisme dans le concept européen de sécurité. On a l'impression que la lutte contre le terrorisme est devenue le nouveau credo des bâtisseurs de l'espace de liberté, de sécurité et de justice. Cette vision des choses est, à maints égards, beaucoup trop manichéenne : la lutte du bien (l'Europe, et plus généralement le monde occidental) contre le mal (le terrorisme et en particulier le terrorisme islamique) est une idée simpliste autour de laquelle il n'est pas possible de construire durablement une politique. En effet, la réaction à une menace n'est pas suffisante pour justifier la construction d'un projet aussi audacieux.

De plus, la focalisation des travaux sur la lutte contre le terrorisme accentue encore plus le déséquilibre dans les relations entre le concept de liberté et celui de la sécurité. Il est évident que la protection de l'Europe est une nécessité, mais il ne faut jamais oublier que la sécurité n'est pas une fin en soi. On ne construit pas l'Europe de la sécurité, on construit l'Europe pour le citoyen.

\section{NOTES}

1. . La « feuille de route» mentionne d'autres aspects de la coopération comme la mise en commun des bonnes pratiques dans le domaine de la lutte contre le terrorisme.

2. . Doc. du Conseil du 20 décembre 2001, n¹5545/01 EUROJUST 15.

3. . Doc. du Conseil du 31 octobre 2001, n¹359/01 LIMITE EUROPOL 82.

4. . Cette clause figurait dans la proposition de la Commission du 22 mars 2002 concernant la signature de l'accord d'association entre la Communauté européenne et l'Algérie (COM (2002) 157 final).

5. . Ce document est disponible sur internet. La feuille de route mise à jour pour le mois de mai est disponible à l'adresse :

http://register.consilium.eu.int/pdf/fr/02/st08/08547f2.pdf

6. . Respectivement : mesure $n^{\circ} 3, n^{\circ} 22, n^{\circ} 49$ et $n^{\circ} 48$.

7. . Communication de la Commission au Conseil, au Parlement européen, au Comité économique et social et au Comité des régions du 26 mai 1999 concernant un Plan 
d'Action de l'Union européenne en matière de lutte contre la drogue (2000-2004) (COM(1999)239 final).

8. . Communication de la Commission au Conseil et au Parlement européen sur un plan d'action de l'Union européenne en matière de lutte contre la drogue (1995-1999) du 26 juin 1994 (COM(94)234).

9. . Ce plan d'action prolonge le programme d'action contre la criminalité organisée qui a été approuvé par le Conseil le 28 avril 1997 (J.O.C.E. C 251 du 15.8.1997, p. 1) et arrivé à échéance.

10. . J.O.C.E. C 142 du 14 juin 2002, p. 23.

11. . Les chefs d'Etat et de gouvernement de l'Union européenne, la présidente du Parlement européen, le président de la Commission européenne, et le Haut représentant pour la politique étrangère et de sécurité commune ont en effet appelé « à la création d'un mandat européen d'arrestation et d'extradition conformément aux conclusions de Tampere, et à la reconnaissance mutuelle des décisions de justice». Quelques jours après, la Commission a présenté officiellement une proposition de décision-cadre ès.

12. . Certains ne disposent pas de législation spécifique sur le terrorisme qui sanctionne les actes terroristes comme des infractions de droit commun. D'autres Etats, à savoir la France, l'Allemagne, l'Italie, le Portugal, l'Espagne, la Grèce et le Royaume-Uni disposent de textes juridiques spécifiques en matière de terrorisme. Cependant dans ces Etats dotés d'une législation spécifique, le terme « terrorisme » recouvre des acceptions différentes.

13. . COM (2001) 521 final.

14. . Décision-cadre du Conseil relative à la lutte contre le terrorisme (J.O.C.E. L 164 du 22 juin 2002, p.3).

15. . Le texte ajoute : « d) le fait de causer des destructions massives à une installation gouvernementale ou publique, à un système de transport, à une infrastructure, $\mathrm{y}$ compris un système informatique, à une plateforme fixe située sur le plateau continental, à un lieu public ou une propriété privée susceptible de mettre en danger des vies humaines ou de produire des pertes économiques considérables ; e) la capture d'aéronefs et de navires ou d'autres moyens de transport collectifs ou de marchandises ; f) la fabrication, la possession, l'acquisition, le transport ou la fourniture ou l'utilisation d'armes à feu, d'explosifs, d'armes nucléaires, biologiques et chimiques ainsi que, pour les armes biologiques et chimiques, la recherche et le développement ; g) la libération de substances dangereuses, ou la provocation d'incendies, d'inondations ou d'explosions, ayant pour effet de mettre en danger des vies humaines ; h) la perturbation ou l'interruption de l'approvisionnement en eau, en électricité ou toute autre ressource naturelle fondamentale ayant pour effet de mettre en danger des vies humaines ; i)la menace de réaliser l'un des comportements énumérés ".

16. . Doc. du Conseil du 13 février 2002, n5712/1/02 REV1 ENFOPOL 18.

17. . Doc du Conseil du 11 mars 2002, n5715/2/02 REV2 ENFOPOL 19.

18. . Initiative du Royaume d'Espagne en vue de l'adoption d'une décision du Conseil relative à l'application de mesures spécifiques de coopération policière et judiciaire en matière de lutte contre le terrorisme, conformément à l'article 4 de la position commune 2001/931/PESC du Conseil (Doc. du Conseil du 18 mars $2002 n^{\circ} 7153 / 02$ CATS 7 RELEX 43). 
19. . Règlement (CE) n 2580/2001 du Conseil du 27 décembre 2001 concernant l'adoption de mesures restrictives spécifiques à l'encontre de certaines personnes et entités dans le cadre de la lutte contre le terrorisme (J.O.C.E. L 344 du 28.12.2001, p. 70). 20. . La liste a fait l'objet d'une mise à jour. Le Conseil a adopté le 17 juin 2002 une décision abrogeant l'ancienne (J.O.C.E. L 10 du 18.06.2002, p. 26).

21. Règlement (CE) n 467/2001 du Conseil interdisant l'exportation de certaines marchandises et de certains services vers l'Afghanistan, renforçant l'interdiction des vols et étendant le gel des fonds et autres ressources financières décidés à l'encontre des Talibans d'Afghanistan, et abrogeant le règlement (CE) n 337/2000 (J.O.C.E. L 67 du 9.03.2001, p.1).

22. . Règlement instituant certaines mesures restrictives spécifiques à l'encontre de certaines personnes et entités liées à Oussama ben Laden, au réseau Al-Qaïda et aux Talibans, et abrogeant le règlement (CE) du Conseil nº67/2001 interdisant l'exportation de certaines marchandises et de certains services vers l'Afghanistan, renforçant l'interdiction des vols et étendant le gel des fonds et autres ressources financières décidées à l'encontre des Talibans d'Afghanistan (J.O.C.E. L 139 du 29 mai 2002, p. 9). Ce Règlement a été modifié par un règlement adopté le 3 juin 2002 (J.O.C.E. L $145 \mathrm{du} 4$ juin 2002, p. 14).

23. . Les groupes terroristes étaient l'émanation de l'idéologie communiste ou anarchiste. C'était le cas d'Action directe en France. Ces groupes terroristes avaient, de près ou de loin, le soutien de Moscou.

24. . Les militaires, qui n'ayant plus à combattre l'ennemi soviétique, développent sur leur territoire les théories de la guerre de «basse intensité ». L'ennemi n'est pas immédiatement identifiable, il est infiltré et se trouve partout.

25. . L'introduction du Programme d'action relatif à la lutte contre la criminalité organisée adopté par le Conseil le 28 avril est très révélatrice :

« Le comportement criminel n'est plus le fait seulement d'individus, mais également d'organisations qui s'insinuent dans les structures de la société civile (...). La criminalité s'organise de plus en plus par delà les frontières nationales tirant également partie de la liberté de circulation (...). Si l'Europe veut devenir un espace de liberté, de sécurité et de justice, elle doit mieux s'organiser et y opposer des réponses stratégiques et tactiques au défi auquel elle est confrontée. (...) Le conseil européen a souligné sa détermination absolue à combattre la criminalité organisée (...) ». (Souligné par l'auteur de l'article).

26. C'est une tautologie d'affirmer que sans personne physique, il n'existerait pas de délinquance. Sans criminel, il n'y aurait pas de crime.

27. . L'ampleur des filières d'immigration clandestine aux mains de la criminalité organisée est devenue un phénomène très préoccupant. La lutte contre ces filières a conduit à la création au sein d'Europol de deux fichiers à des fins d'analyse sur l'immigration clandestine (Rapport annuel d'activité d'Europol, 1999, p. 11).

28. . X. RAUFER et S. QUERE., Le crime organisé, coll. Que sais-je ?, PUF, 1999, p. 31. 29. . J.-Y. CARLIER et F. CREPEAU, « Intégration régionale et politique migratoire - Le « modèle » européen entre coopération et communautarisation », Journal de Droit international, 1999, p. 1016.

30. . Adoptées par le Conseil « Justice, affaires intérieures et protection civile » des 25 et 26 avril 2002 (7991/01 (Presse 104)).

31. . Juste après les attentats, les médias du monde entier ont montré de manière quasicontinue pendant plusieurs heures la scène montrant des avions s'écrasant contre les 
tours. Ils ont également évoqué leur effondrement des heures durant pendant les semaines qui suivirent. Cet impact a créé un émoi très grand dans la population, voire une véritable psychose (il suffit de voir l'effet des attentats sur le chiffre d'affaire des compagnies aériennes).

32. . H. LABAYLE, La réforme du troisième pilier, Contribution au nom du Mouvement européen France-Groupe troisième pilier pour la Convention sur l'avenir de l'Union, non publiée, 2002, p. 1.

33. . Le mandat d'arrêt européen a été adopté en quatre mois. A titre de comparaison, la Convention d'entraide judiciaire a mis des années à voir le jour. Le premier projet a été élaboré sous présidence italienne du premier semestre 1996. Le programme d'action sur la criminalité organisée adopté par le Conseil européen d'Amsterdam, prévoyait qu'une nouvelle convention d'entraide judiciaire devait être signée « avant fin 1997/ mi-1998 ». Finalement, la Convention a été signée le 29 mai 2000.

34. . COM (2000) 624 du 10 octobre 2001 : Proposition modifiée de directive relative au droit au regroupement familial.

35. . COM (2000) 854(01) du 22 janvier 2001.

36. . Initiative du Royaume-Uni, de la République française et du Royaume de Suède en vue de l'adoption par le Conseil d'une décision-cadre concernant l'application du principe de reconnaissance mutuelle aux sanctions pécuniaires (J.O.C.E. C $278 \mathrm{du}$ 2.10.2001 p. 4).

\section{INDEX}

Index chronologique : 2001 post 11 septembre

Mots-clés : construction européenne, droit européen, libertés publiques, Politique européenne de voisinage, sécurité

Thèmes : EUROJUST, EUROPOL

Index géographique: Union européenne 